\title{
Comparative Evaluation of Hormones and Hormone-Like Molecule in Lineage Specification of Human Induced Pluripotent Stem Cells
}

\author{
Seon-A Choi ${ }^{1,2, *}$, Ju-Hyun An ${ }^{1,2,3, *}$, Seung Hwan Lee ${ }^{1,2, *}$, Geun-Hui Lee ${ }^{1,2}$, Hae-Jun Yang ${ }^{1,2}$, Pil-Soo Jeong ${ }^{1,2}$, \\ Jae-Jin Cha $^{1,2}$, Sanghoon Lee ${ }^{1,2}$, Young-Ho Park ${ }^{1,2,3}$, Bong-Seok Song ${ }^{1,2,3}$, Bo-Woong Sim ${ }^{1,2}$, Young-Hyun Kim ${ }^{2,3}$, \\ Ji-Su Kim ${ }^{2,3,4}$, Yeung Bae Jin ${ }^{2}$, Jae-Won Huh ${ }^{2,3}$, Sang-Rae Lee ${ }^{2,3}$, Jong-Hee Lee ${ }^{1,2}$, Sun-Uk Kim ${ }^{1,2,3}$ \\ ${ }^{I}$ Futuristic Animal Resource E Research Center (FARRC), \\ Korea Research Institute of Bioscience and Biotechnology (KRIBB), Cheongiu, Korea \\ ${ }^{2}$ National Primate Research Center (NPRC), Korea Research Institute of Bioscience and Biotechnology (KRIBB), Cheongju, Korea \\ ${ }^{3}$ Department of Functional Genomics, KRIBB School of Bioscience, Korea University of Science and Technology (UST), Daejeon, Korea \\ ${ }^{4}$ Primate Resource Center, Korea Research Institute of Bioscience and Biotechnology, Feongeup, Korea
}

Background and Objectives: Proficient differentiation of human pluripotent stem cells (hPSCs) into specific lineages is required for applications in regenerative medicine. A growing amount of evidences had implicated hormones and hormone-like molecules as critical regulators of proliferation and lineage specification during in vivo development. Therefore, a deeper understanding of the hormones and hormone-like molecules involved in cell fate decisions is critical for efficient and controlled differentiation of hPSCs into specific lineages. Thus, we functionally and quantitatively compared the effects of diverse hormones (estradiol 17- $\beta$ (E2), progesterone (P4), and dexamethasone (DM)) and a hormone-like molecule (retinoic acid (RA)) on the regulation of hematopoietic and neural lineage specification. Methods and Results: We used $10 \mathrm{nM} \mathrm{E2,} 3 \mu \mathrm{M} \mathrm{P} 4,10 \mathrm{nM}$ DM, and $10 \mathrm{nM}$ RA based on their functional in vivo developmental potential. The sex hormone E2 enhanced functional activity of hematopoietic progenitors compared to P4 and DM, whereas RA impaired hematopoietic differentiation. In addition, E2 increased CD $34^{+} \mathrm{CD} 45^{+}$cells with progenitor functions, even in the $\mathrm{CD} 43^{-}$population, a well-known hemogenic marker. RA exhibited lineage-biased potential, preferentially committing hPSCs toward the neural lineage while restricting the hematopoietic fate decision. Conclusions: Our findings reveal unique cell fate potentials of E2 and RA treatment and provide valuable differentiation information that is essential for hPSC applications.

Keywords: Human induced pluripotent stem cells, lineage specification, Estradiol-17 $\beta$, Retinoic acid, Hematopoietic differentiation, Cell fate decision

Received: December 24, 2018, Revised: May 10, 2019, Accepted: May 25, 2019, Published online: June 30, 2019

Correspondence to Jong-Hee Lee

Futuristic Animal Resource \& Research Center (FARRC), Korea Research Institute of Bioscience and Biotechnology (KRIBB), 30 Yeongudanji-ro, Cheongwon-gu, Cheongju 28116, Korea

Tel: +82-43-240-6312, Fax: +82-43-240-6309, E-mail: jonglee@kribb.re.kr

Co-Correspondence to Sun-Uk Kim

Futuristic Animal Resource \& Research Center (FARRC), Korea Research Institute of Bioscience and Biotechnology (KRIBB), 30 Yeongudanji-ro, Cheongwon-gu, Cheongju 28116, Korea

Tel: +82-43-240-6321, Fax: +82-43-240-6309, E-mail: sunuk@kribb.re.kr

${ }^{*}$ These authors contributed equally to this work.

(a) This is an open-access article distributed under the terms of the Creative Commons Attribution Non-Commercial License (http://creativecommons.org/licenses/by-nc/4.0/), which permits unrestricted non-commercial use, distribution, and reproduction in any medium, provided the original work is properly cited.

Copyright (c) 2019 by the Korean Society for Stem Cell Research 


\section{Introduction}

The extraordinary potential of human pluripotent stem cells (hPSCs), including unlimited self-renewal and capacity for differentiation, has provided new surrogate models to understand the fundamental human developmental progression and regenerative therapeutics. However, an understanding of complex differentiation processes is required to instruct proficient lineage specifications for future clinical applications. Hematopoietic stem cells (HSCs) and neural stem cells (NSCs) replenish whole blood and the nervous system in the human body, respectively, and have recently emerged as the most applicable derivatives of hPSCs. Despite a considerable number of reports demonstrating distinct intrinsic and extrinsic factors affecting lineage specification and self-renewal (1) and deriving in vivo counterparts from hPSCs (2), the differentiation programs are complex and remain to be completely elucidated.

Developmental and functional regulation and homeostasis of the hematopoietic system and in vivo neural systems are tightly controlled by various physiological characteristics, including hormone fluctuations and responses to inflammatory stress (reviewed in 3). Hormones are signaling molecules produced by the endocrine system that contribute to physiological regulation and behavioral activities. Recent studies have demonstrated the critical roles of hormones in lineage specification and maturation of multipotent and pluripotent stem cells. Estradiol 17- $\beta$ (E2), a major endogenous steroid hormone, exerts various biological effects, such as maturation and functions of reproductive organs, bone formation, and lipid metabolism. Moreover, recent studies have provided new evidence of the enhanced self-renewal of HSCs and their differentiation into megakaryocytes and erythroid progenitors (4). Estrogen receptor (ER) $\beta$ is also implicated in the proliferation and self-renewal of NSCs through Notch signaling, and dominates dopaminergic fate decisions via prevention of oligodendrogliogenesis (5). Progesterone (P4), another important steroid sex hormone that coordinates pregnancy and sexual organ development in females in addition to E2, is reportedly associated with mammary stem cells and breast cancer stem cells (6). Aside from its role in the reproductive system, a recent study suggested a new functional role of $\mathrm{P} 4$ in the regulation of the regenerative potential of amniotic epithelial cells by preventing the epithelial-mesenchymal transition (7).

Glucocorticoids (GCs), involved in the immune response and glucose metabolism, are also required for fetal organ maturation. For instance, administration of dex- amethasone (DM), a synthetic GC, altered hypothalamic development and increased the expression of genes related to self-renewal and progenitor functions in embryonic hypothalamic NSCs. In addition, GCs synthesized in neural progenitor cells (NPCs) derived from hPSCs could maintain self-renewal of NPCs by suppressing neuronal differentiation (8). A recent study also revealed that DM increased the colony-forming unit (CFU)-erythroid of HSCs in vitro, thereby reducing transfusion dependency in myelodysplastic syndrome patients (9).

Retinoic acid (RA) is a morphogen essential for embryonic development by regulating differentiation and homeostasis of diverse cell lineages in a pleiotropic manner (10). RA is an important factor for induction of PSC differentiation, especially into pancreatic and neural lineages at high concentrations $(11,12)$. For example, RA can promote efficient generation of specific types of neurons, including GABAergic (13), dorsal spinal sensory interneuron (14), dopaminergic, and striatopallidal medium spiny neurons (15). Although their involvement in hematopoietic differentiation during developmental stages has not been well reported, recent studies have indicated that RA signaling is required to maintain homeostasis of the hematopoietic system $(16,17)$. Recently, it was also reported that RA regulated the generation of hematopoietic progenitors from hPSCs in a concentration- and time-dependent manner (18).

Although several studies have demonstrated the crucial roles of hormones and hormone-like molecules in lineage-specific differentiation of hPSCs, clear functional and quantitative comparisons of each molecule in terms of their fate decisions, especially in hematopoietic and neural specification, have not yet been provided. In this study, using human induced PSCs (hiPSCs) derived from human $\mathrm{CD} 34^{+}$hematopoietic progenitors, we newly identified the distinct cell-fate potential of hormones (E2, P4, and DM) and a hormone-like molecule (RA). E2 signaling enriched the hematopoietic differentiation potentials of hPSCs without perturbing fate decisions into other cell types. However, RA distinctly suppressed hematopoietic specification and functional activity of generated progenitors, which was compensated by enhanced neural differentiation. Taken together, our data provide new evidence that E2 and RA tightly control differentiation potential in two distinct manners, potentiation of lineage specification and biased cell fate by compromising other lineage decisions. 


\section{Materials and Methods}

\section{Cell culture, hiPSC generation, and lineage specification}

To obtain hiPSCs, $\mathrm{CD} 34^{+}$cells were purified from primary human bone marrow (BM) cells (Allcells, Emeryville, CA, USA) via immunomagnetic separation (human CD34 microbeads; Miltenyi Biotec, Bergisch Gladbach, Germany). Isolated $\mathrm{CD} 34^{+} \mathrm{BM}$ cells were transduced with OCT3/4, SOX2, KLF4, and c-Myc sendai virus (Cytotune 2.0; Thermo Fisher Scientific, Waltham, MA, USA) in Iscove's modified Dulbecco's medium (Gibco) supplemented with $15 \%$ bovine serum albumin, $1 \times$ BIT (Stemcell Technologies, Vancouver, Canada), 1\% non-essential amino acids (Gibco), $100 \mathrm{ng} / \mathrm{mL}$ stem cell factor, $100 \mathrm{ng} / \mathrm{mL}$ thrombopoietin, $100 \mathrm{ng} / \mathrm{mL}$ Flt-3 ligand, and $20 \mathrm{ng} / \mathrm{mL}$ interleukin-3 (R\&D Systems, Minneapolis, $\mathrm{MN}$, USA) as previously described $(19,20)$. After a $48 \mathrm{~h}$ incubation, transduced $\mathrm{CD} 34^{+} \mathrm{BM}$ cells were maintained on matrigel (BD Biosciences, Franklin Lakes, NJ, USA)-coated plates with TeSR-E8 iPSC culture medium (Stemcell Technologies). hiPSC colonies were manually selected $\sim 15$ days after transduction and expanded in TeSR-E8 medium.

\section{Hematopoietic differentiation}

For hematopoietic differentiation from hPSCs, single cells were aggregated as spin embryoid bodies (EBs) as described previously (21) with modifications. In brief, hPSCs were dissociated as single cells with accutase (SigmaAldrich, St. Louis, MO, USA), plated at 10,000 cells in $50 \mu \mathrm{L}$ medium per well of a round-bottom low-attachment 96-well plate (Corning) in TeSR-E8 supplemented with Y-27632 (i.e., ROCK inhibitor), and then centrifuged for $5 \mathrm{~min}$ at $1,500 \mathrm{rpm}$ (day -1). After $24 \mathrm{~h}$, EBs were transferred to STEMdiff ${ }^{\mathrm{TM}}$ hematopoietic differentiation medium (Stemcell Technologies) according to the manufacturer's protocol (day 0). The medium of spin EBs was changed on day 3 , after which the cells were incubated until day 12 for the hematopoietic analysis.

\section{CFU (colony-forming unit) assay}

A CFU assay was performed by plating a single-cell suspension of human embryoid bodies (hEBs) in methylcellulose SF H4436 medium (Stemcell Technologies). hEBs were dissociated into single cells, filtered through a $40-\mu \mathrm{m}$ cell strainer, and then $5 \times 10^{3}$ cells in methylcellulose SF H4436 medium were plated in 12-well plates (BD Falcon). For characterization of hematopoietic progenitor potential, different colony counts were performed based on morphology after 12 14 days.

\section{Neural differentiation}

For neural differentiation, spin EBs were generated by plating $2 \sim 3 \times 10^{4}$ single cells in $100 \mu \mathrm{L}$ per well of a round-bottom low-attachment 96 -well plate in neural differentiation medium (DMEM:F12 (1: 1 ; Thermo Fisher Scientific) supplemented with N2 (Thermo Fisher Scientific), B27 (Thermo Fisher Scientific), SB431542 (10 $\mu \mathrm{M}$; Sigma-Aldrich), LDN193189 (100 nM; Sigma-Aldrich)), and then centrifuged for $5 \mathrm{~min}$ at 1,500 rpm. EBs were cultured for $7 \sim 10$ days, with media changes every other day. Neural precursors were analyzed by dissociating EBs into single cells and performing flow cytometry with the NSC markers NESTIN and PAX6 (R\&D Systems).

\section{RNA isolation and real-time PCR}

Total RNA was isolated and purified using the RNeasy Kit (Qiagen, Hilden, Germany) following the manufacturer's protocol. Human embryonic stem cell H9 (H9 ESCs) total RNA was purchased from Sciencell. cDNA was synthesized using the ReverTra Ace-cDNA synthesis kit (Toyobo, Osaka, Japan), and subsequent quantitative real-time PCR was performed using Takara SYBR Green PCR Master Mix (Applied Biosystems, Foster City, CA, USA). Amplifications were executed in triplicate and the relative expression of genes was calculated using the $\Delta \Delta$ Ct method, as reported previously (19). PCR was carried out using the following conditions: $95^{\circ} \mathrm{C}$ for $10 \mathrm{~min}$, followed by 40 cycles of $95^{\circ} \mathrm{C}$ for $15 \mathrm{~s}$, and $60^{\circ} \mathrm{C}$ for $1 \mathrm{~min}$. The primers used for real-time PCR were in Table 1. All data were normalized to GAPDH levels.

\section{Immunocytochemistry and imaging}

Cells were fixed in BD Cytofix ${ }^{\mathrm{TM}}$ fixation buffer (BD Biosciences) for $15 \mathrm{~min}$ at room temperature and then washed three times with BD perm/wash buffer (BD Biosciences) every $10 \mathrm{~min}$. After a $30 \mathrm{~min}$ incubation with perm/wash buffer at room temperature, cells were stained with the following conjugated primary antibodies and incubated overnight at $4^{\circ} \mathrm{C}$ : OCT3/4-FITC $(1: 100$; BD Pharmingen), NANOG-PE ( $1: 100$; BD Pharmingen), SOX2-PE (1: 50; BD Biosciences), SSEA3-PE (1:100; BD Pharmingen), SSEA4-FITC (1 : 100; BD Biosciences), TRA-1-60-FITC (1 : 100; BD Pharmingen), and $\beta$ III-tubulin-FITC (1 : 100; BD Biosciences). Cells were imaged using fluorescence microscopy (Leica, Wetzlar, Germany).

\section{Flow cytometry}

Single-cell suspensions were stained with fluorochromeconjugated antibody to evaluate pluripotency and hematopoietic and neural differentiation. Briefly, cells were dis- 
Table 1. Sequences of primers used for quantitative real-time PCR

\begin{tabular}{lll}
\hline & & \multicolumn{1}{c}{ Primer sequences } \\
\cline { 2 - 3 } Target genes & \multicolumn{1}{c}{ Forward } & Reverse \\
\cline { 2 - 3 } NCT3/4 & TGCCTTTTAAAATCCAGTCCCA & ACCTTCCCAAATAGAACCCCC \\
SOX2 & AACAGGTGAAGACCTGGTTCC & CTGAGGCCTTCTGCGTCACA \\
SCLTAL1 & GGATAAGTACACGCTGCCCG & ATGTGCGCGTAACTGTCCAT \\
GATA2 & CATGGTGCAGCTGAGTCCT & CCATCTCATAGGGGGAAGGT \\
C/EBP1 $\alpha$ & GGGCTAGGGAACAGATGGACG & GCAGCAGTCAGGTGCGGAGG \\
BRACHYURY & CTAGAGATCTGGCTGTGGGG & TCATAACTCCGGTCCCTCTG \\
MIXL1 & ATGAGCCTCGAATCCACATAGT & TCCTCGTTCTGATAAGCAGTCA \\
PAX6 & GGATCCAGGTATGGTTCCAG & GGAGCACAGTGGTTGAGGAT \\
NF68 & CCGGCAGAAGATTGTAGAGC & CGTTGGACACGTTTGATTG \\
NeuroD & CAGACCGAAGTGGAGGAAAC & CCTCTTCCTTGTCCTTCTCCT \\
GAPDH & TGAAAGCCCTCTGACTGATTGC & AGCGGTGCTGAGAAGATTGA \\
& TGCACCACCAACTGCTTAGC & GGCATGGACTGTGGTCATGAG
\end{tabular}

sociated with cell dissociation buffer (Gibco) for $10 \mathrm{~min}$, and then resuspended in phosphate-buffered saline supplemented with $3 \% \mathrm{v} / \mathrm{v}$ fetal bovine serum. The following antibodies were used for visualization: CD34-PE (1 : 100; BD Biosciences), CD43-FITC (1 : 100; BD Biosciences), CD45-APC (1 : 100; BD Biosciences), PAX6-PE (1 : 100; BD Biosciences), NESTIN-FITC (1:100; Millipore, Burlington, MA, USA), and LIVE/DEAD-APC-Cy7 (1 : 7500; Thermo Fisher Scientific). Fluorescence activated cell sorting (FACS) analysis was performed with a BD FACSAria $^{\mathrm{TM}}$ III (BD Biosciences), and all data were analyzed with FlowJo software (Tree Star, Ashland, OR, USA).

\section{Statistical analysis}

All data were expressed as the means and standard deviation (SD) of at least three independent experiments. Experimental differences were evaluated for statistical significance using Prism software (GraphPad Software). Statistical significance was determined using an unpaired Student's $t$-test, and the results were considered significant or highly significant when $\mathrm{p}<0.05$ or $\mathrm{p}<0.01$, respectively.

\section{Results}

\section{Derivation and characterization of hiPSCs}

To assess the functional roles of hormones and hormone-like molecules in developmental regulation, we first generated iPSCs from CD34 ${ }^{+}$adult BM mononuclear cells (MNCs). iPSC lines were derived via transduction of $\mathrm{CD}^{+}{ }^{+}$BM MNCs with Oct4-, Sox2-, c-Myc-, and Klf4-expressing sendai virus. The morphology of the gen- erated iPSC line was indistinguishable from hPSCs (Fig. 1A). iPSCs were evaluated for a fully reprogrammed state using immunocytochemical analysis for expression of pluripotent markers; SSEA3, SSEA4, TRA-1-60, SOX2, NANOG and OCT3/4 (Fig. 1B). FACS analysis of the aforementioned markers further indicated that iPSCs derived from $\mathrm{CD}_{34}{ }^{+} \mathrm{BM}$ MNCs were not distinguishable from typical hPSCs (Fig. 1C). Karyotype analysis revealed a normal $44+\mathrm{XX}$ chromosomal number and appearance, suggesting that genomic integrity was not compromised in these iPSCs (Fig. 1D). Finally, we confirmed the expression of pluripotent genes of generated iPSCs via real-time PCR (Fig. 1E). The results revealed that the iPSCs were equivalent to conventional hPSCs using routinely applied criteria to identify the fully established human pluripotent state.

\section{Effects of hormones and hormone-like molecules on hematopoietic differentiation potential}

To assess the functional roles of hormones and in controlled lineage specification, we first evaluated in vitro hematopoietic potential using hEB-based differentiation methods, as described previously (Fig. 2A) (22). We used $10 \mathrm{nM} \mathrm{E2,} 3 \mu \mathrm{M} \mathrm{P} 4,10 \mathrm{nM} \mathrm{DM}$, and $10 \mathrm{nM}$ RA based on their functional in vivo developmental potential. Optimized hematopoietic conditions resulted in a dramatic increase in hematopoietic cells $\left(\mathrm{CD} 45^{+}\right)$and progenitors $\left(\mathrm{CD} 34^{+} \mathrm{CD} 45^{+}\right)$and time-dependent upregulation of mesodermal (BRACHYURY and MIXL1) and hematopoietic (C/EBP1 $\alpha$, RUNX1, GATA2 and SCL/TAL1) lineage markers consistent with hematopoietic differentiation (Supplementary Fig. S1A). Under such conditions, the hormones had no effect on the output of hem- 
A

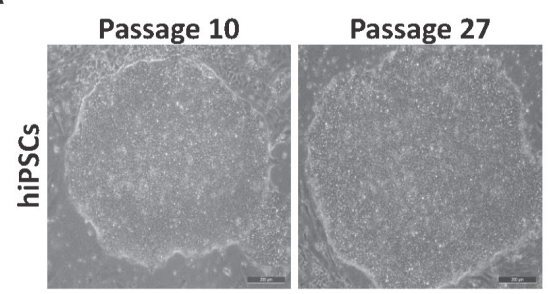

C
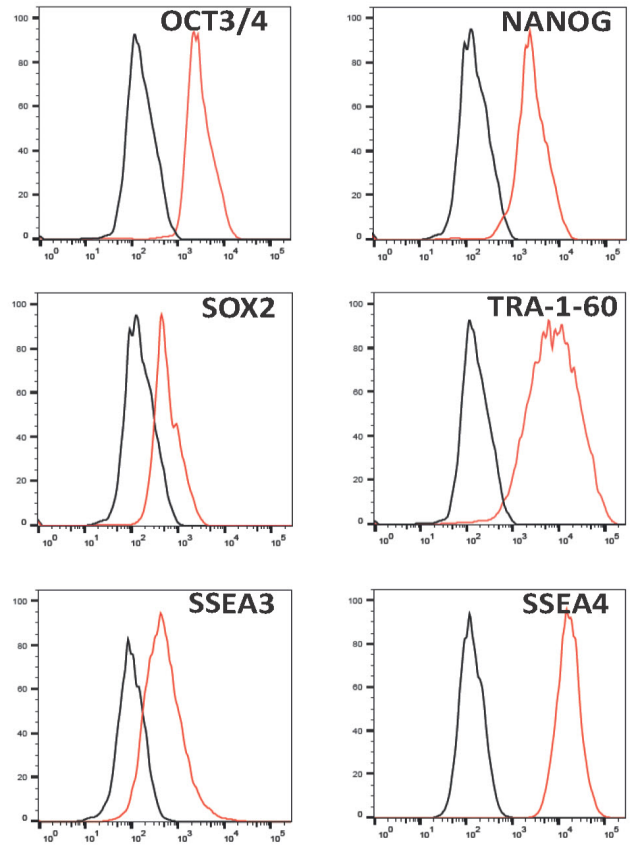

- Isotype

— hiPSCs
B

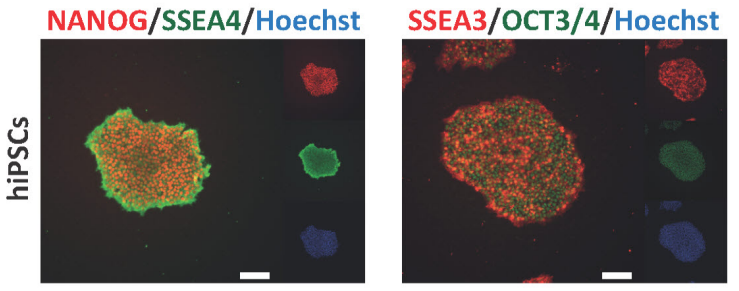

SOX2/TRA-1-60/Hoechst

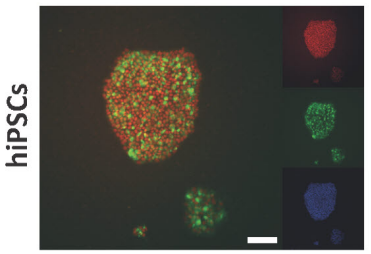

E

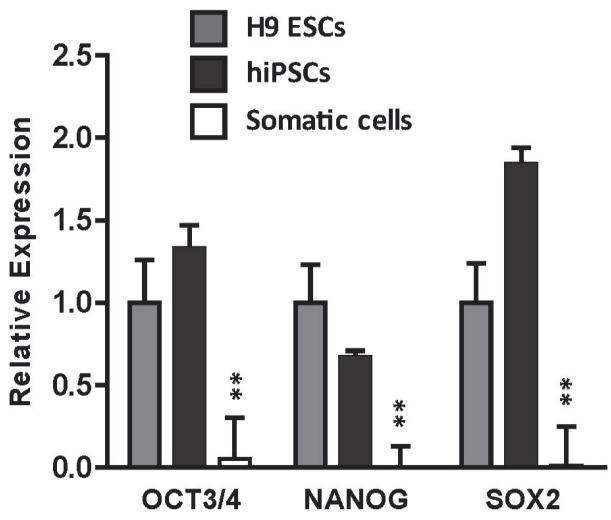

D

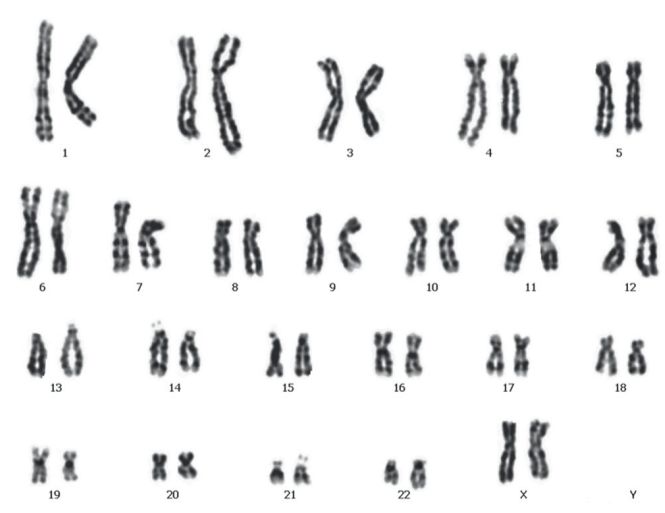

Fig. 1. Derivation of hiPSCs from BM CD34 ${ }^{+}$cells. (A) Representative phase-contrast image of hiPSCs. hiPSCs were characterized by embryonic stem cell morphology. Scale bar, $200 \mu \mathrm{m}$. (B) Immunocytochemical analysis of pluripotent markers. Representative images of hiPSCs stained with the pluripotent markers OCT3/4, NANOG, SOX2, SSEA3, SSEA4 and TRA-1-60. Scale bar, $200 \mu$ m. Nuclei were stained with hoechst33342 (blue). (C) Flow cytometric analysis of pluripotent marker expression. Black histogram bars indicate negative isotype controls. (D) Karyotype analysis of hiPSCs, displaying a normal 44+XX karyotype. (E) Quantitative real-time PCR analysis of pluripotent genes in hiPSCs normalized to GAPDH. \#Data are presents as the means \pm SD of three independent experiments. ${ }^{*} p<0.05 ; * * p<0.01$. 
A

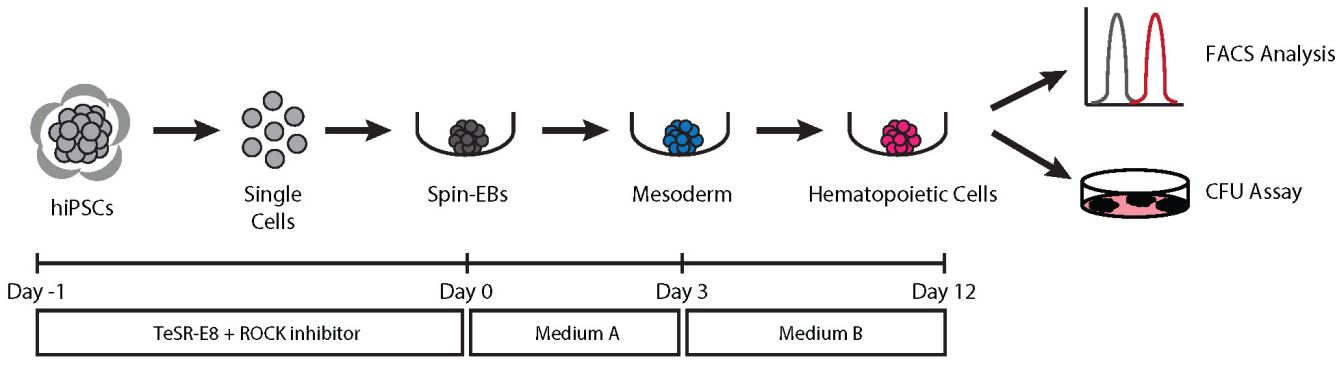

B

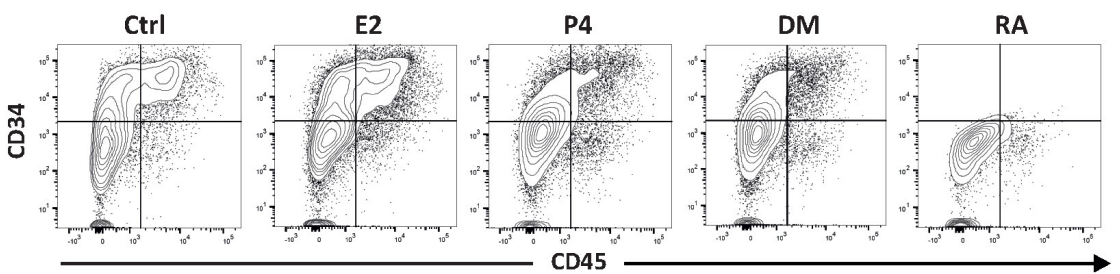

C

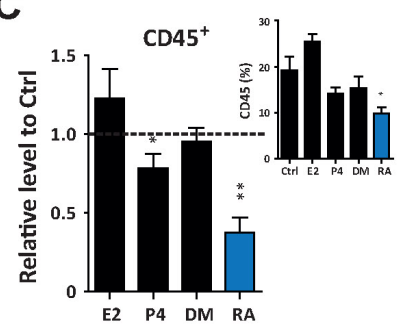

D

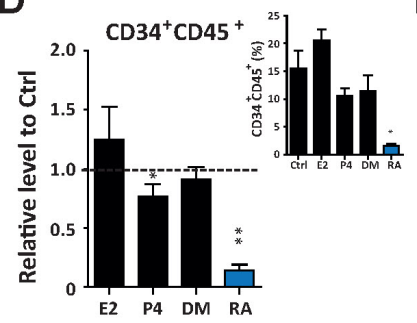

H

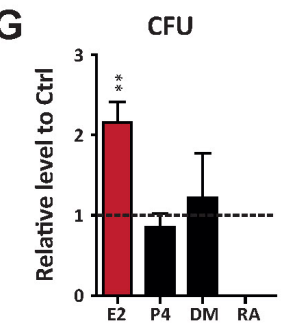

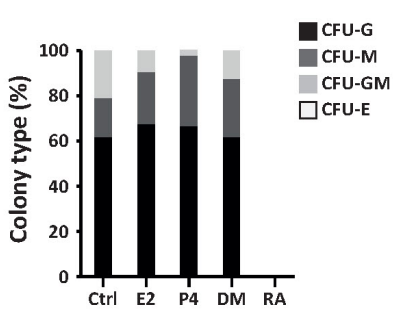

E

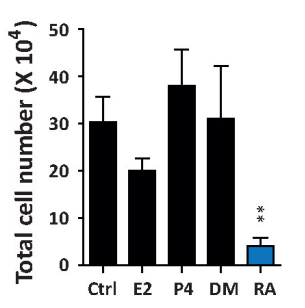

F

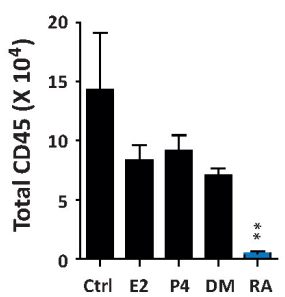

I

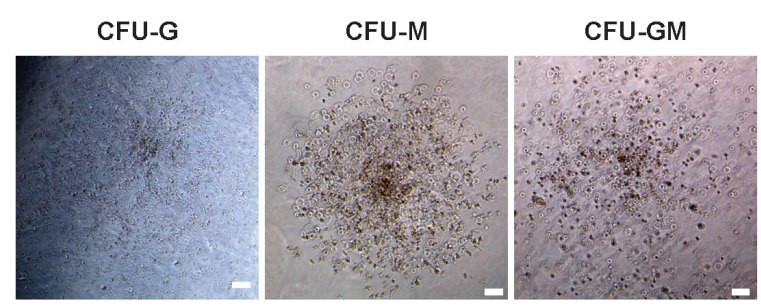

Fig. 2. Distinct hematopoietic differentiation potential of hormones and hormone-like molecule. (A) Schematic protocol for hematopoietic differentiation of hPSCs. (B F) Effects of hormones (E2, P4, and DM) and a hormone-like molecule (RA) on the production of hematopoietic cells $\left(\mathrm{CD} 45^{+}\right)$and progenitors $\left(\mathrm{CD} 34^{+} \mathrm{CD} 45^{+}\right)$. Ctrl: control, E2: estradiol-17 $\beta$, P4: progesterone, DM: dexamethasone. (B) Flow cytometric plots showing hematopoietic cells $\left(\mathrm{CD} 45^{+}\right)$and progenitors $\left(\mathrm{CD} 34^{+} \mathrm{CD} 45^{+}\right)$at day 12 following hematopoietic differentiation with or without E2, P4, DM, and RA treatment. (C) Relative levels of $\mathrm{CD}_{4} 5^{+}$cells normalized to the control. The inset shows the percentage of $\mathrm{CD} 45^{+}$ cells. (D) Relative levels of hematopoietic progenitors $\left(\mathrm{CD} 34^{+} \mathrm{CD} 45^{+}\right)$normalized to the control. The inset indicates the percentage of $\mathrm{CD} 34^{+} \mathrm{CD} 45^{+}$cells. Number of (E) total cells and (F) $\mathrm{CD} 45^{+}$cells during hematopoietic differentiation with or without treatment with hormones and hormone-like molecule. (G) Total number of hematopoietic CFUs generated from day $12 \mathrm{hEBs}$. (H) Distribution of colony subtypes derived from day $12 \mathrm{hEBs}$. (I) Representative CFU morphology. Scale bar, $100 \mu \mathrm{m}$. CFU-granulocytes (CFU-G); CFU-macrophages (CFU-M); CFU-granulocytes/macrophages (CFU-GM); CFU-erythroid (CFU-E). \#Data are presents as the means \pm SD of three or six independent experiments. ${ }^{*} \mathrm{p}<0.05 ; * * \mathrm{p}<0.01$.

atopoietic cells, including progenitors. However, RA highly suppressed hematopoietic differentiation, drastically limiting the number of hematopoietic cells and progenitors (Fig. $2 \mathrm{~B} \sim \mathrm{D}$ ). In addition, cells under hematopoietic conditions were significantly less proliferative after RA treatment. Consequently, the overall number of hematopoietic cells was highly restricted (Fig. 2E, 2F). To further compare the multi-lineage potential of hematopoietic progenitors under different conditions, we performed hematopoietic CFU assays. Consistent with its limited po- 
tential to generate hematopoietic cells, RA impaired production of CFUs from progenitors. Interestingly, E2 significantly increased the total number of CFUs produced by progenitors compared to other hormones, suggesting a critical influence of E2 on the functional capacity of hematopoietic progenitors (Fig. 2G). Increase of mesodermal (BRACHYURY and MIXL1) and hematopoietic (C/EBP1 $\alpha$, RUNX1, GATA2 and SCL/TAL1) lineage markers upon E2 treatment further supports the effect of E2 on hematopoietic differentiation of hPSCs (Supplementary Fig. S1B). However, the distribution of CFU types was not affected, suggesting no preferential blood lineage specification by the investigated hormones (Fig. 2H, 2I). Taken together, these results demonstrate that $\mathrm{E} 2$ potentiates the functional ability of generated hematopoietic progenitors, whereas RA restricts hematopoietic specification.

\section{E2 enhances the functional ability of hemogenic precursors}

CD43, also known as leukosialin or sialoglycoprotein, has been reported as a hemogenic marker (23). CD43 is expressed in early hematopoietic cells differentiated from hPSCs, and marks multipotent hematopoietic progenitors. Consistent with the hematopoietic potential of hormones and hormone-like molecules, CD43 expression was significantly down-regulated after RA treatment (Fig. 3A). Given that CD43 defines hematopoietic lineages, most hematopoietic cells $\left(\mathrm{CD} 45^{+}\right)$, including progenitors $\left(\mathrm{CD} 34^{+} \mathrm{CD} 45^{+}\right)$, are positive for CD43 expression (Fig. $3 \mathrm{~A} \sim \mathrm{C})$. However, we newly identified $\mathrm{CD} 34^{+} \mathrm{CD} 45^{+}$ hematopoietic progenitors in the $\mathrm{CD}^{-} 3^{-}$population, which were produced in response to E2 (Fig. 3D). To further evaluate their functional ability as progenitors, $\mathrm{CD} 43^{-}$ $\mathrm{CD} 34^{+} \mathrm{CD} 45^{+}$cells were isolated by FACS and subsequently applied to the in vitro CFU assay. Sorted cells gave rise to terminally differentiated granulocytic and monocytic lineages (Fig. 3E). These results further confirm the regulatory role of E2 signaling in hematopoietic fate decisions of hPSCs and demonstrate new possible hematopoietic cells $\left(\mathrm{CD} 43^{-} \mathrm{CD} 34^{+} \mathrm{CD} 45^{+}\right)$from $\mathrm{CD} 43$

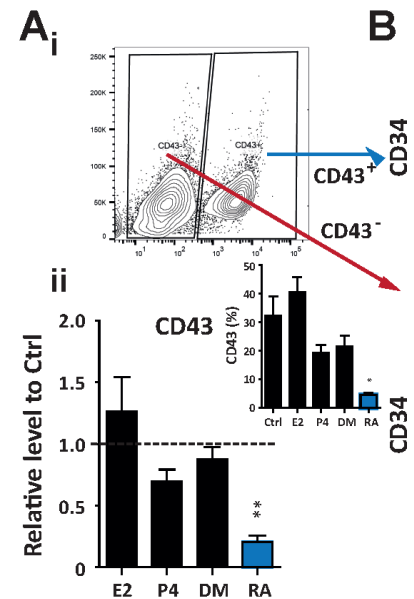

C

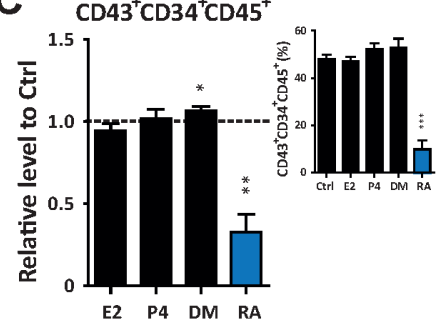

B
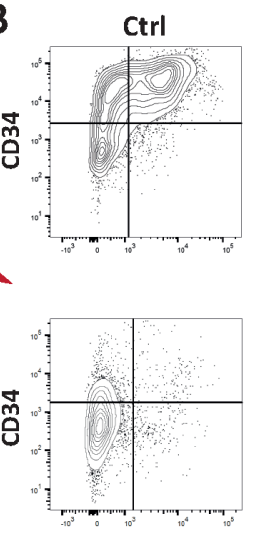

D

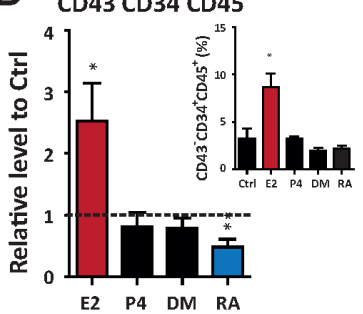

E2
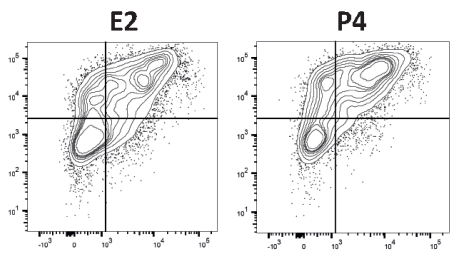

CD45

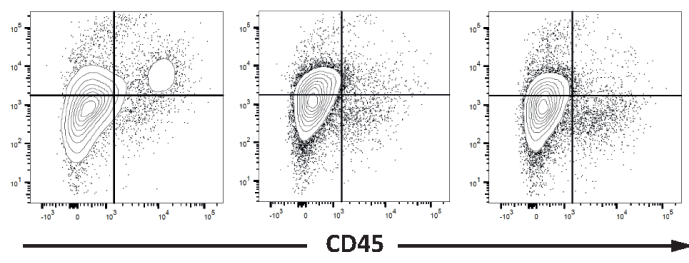

$\mathbf{E}$

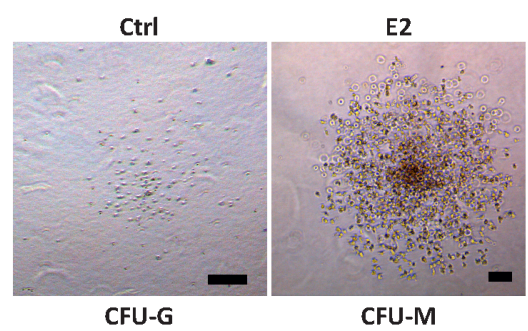

Fig. 3. E2 enhances the functional ability of hemogenic precursors. (A) i. Flow cytometric analysis identifying the CD43 ${ }^{+}$population during hematopoietic differentiation. ii. Relative levels of $\mathrm{CD} 43^{+}$cells normalized to the control. The inset shows the percentage of $\mathrm{CD} 43^{+}$cells. (B) Flow cytometry gating of the $\mathrm{CD}_{3} 3^{+}$and $\mathrm{CD} 43^{-}$fraction to analyze the frequency of hematopoietic progenitors $\left(\mathrm{CD} 34^{+} \mathrm{CD} 45^{+}\right) .(\mathrm{C})$ Relative proportions of hematopoietic progenitors of $\mathrm{CD}_{3} 3^{+}$fractions normalized to the control at day 12 . The inset represents the percentage of $\mathrm{CD} 34^{+} \mathrm{CD} 45^{+}$cells. (D) Relative levels of $\mathrm{CD} 34^{+} \mathrm{CD} 45^{+}$cells gated from the $\mathrm{CD} 43^{-}$population normalized to the control. The inset displays the percentage of $\mathrm{CD} 34^{+} \mathrm{CD} 45^{+}$cells. (E) Representative CFU morphologies derived from the $\mathrm{CD} 43^{-} \mathrm{CD} 34^{+} \mathrm{CD} 45^{+}$population treated with E2. Scale bar, $100 \mu \mathrm{m}$. \#Data are presents as the means \pm SD of three independent experiments. ${ }^{*} \mathrm{p}<0.05 ;{ }^{* *} \mathrm{p}<0.01$. 
A

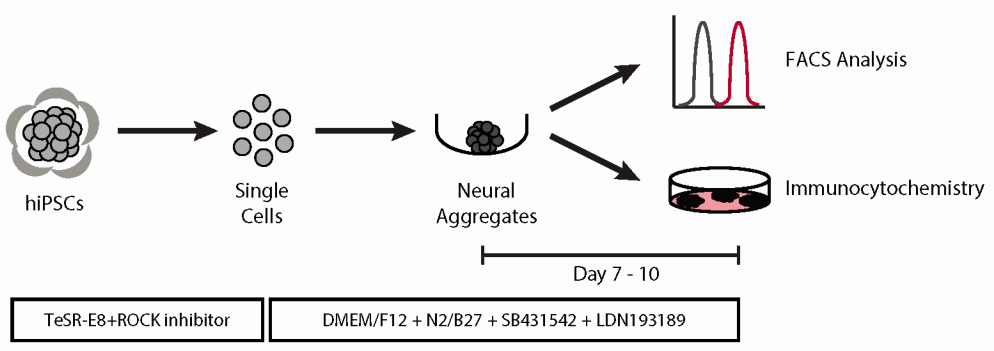

B
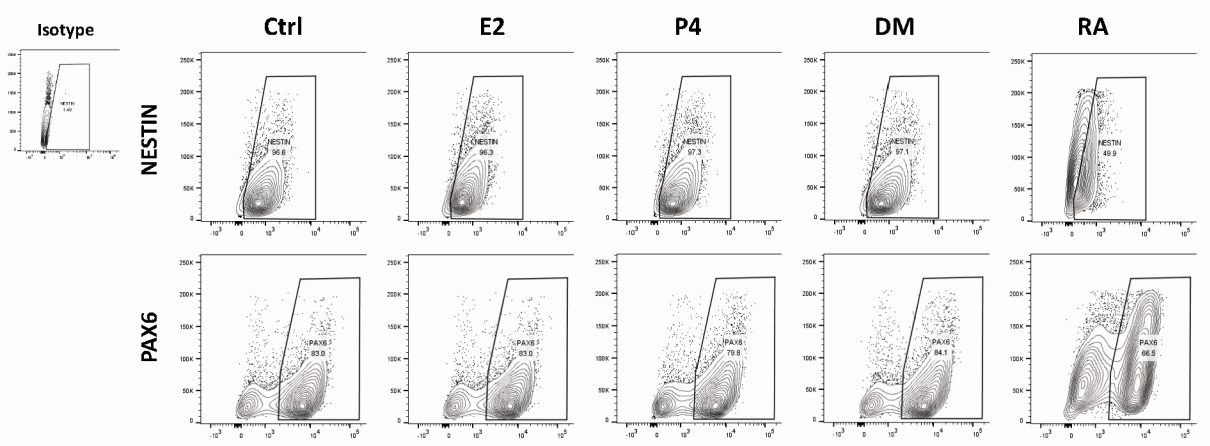

C
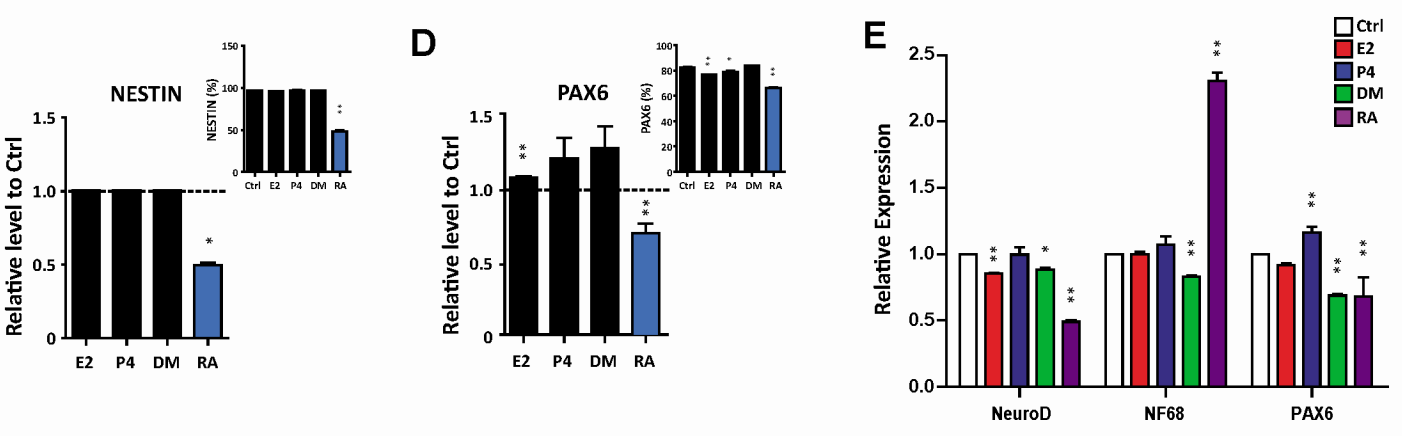

$\mathbf{F}$

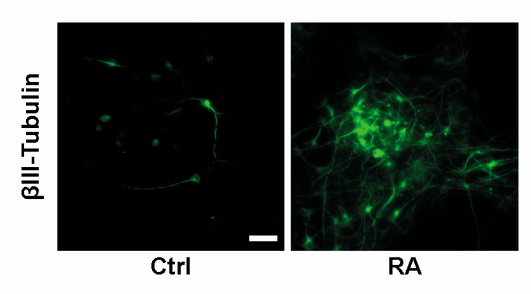

G

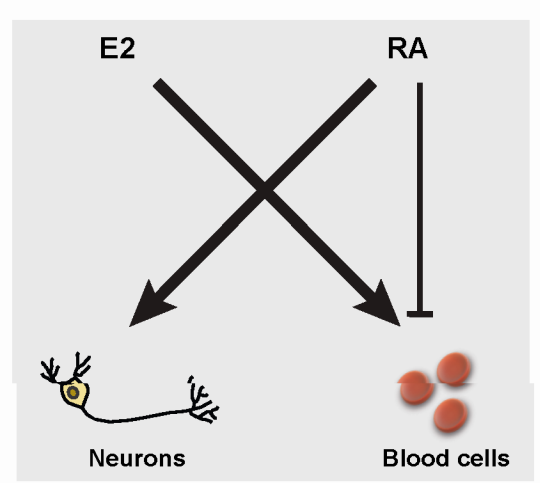

Fig. 4. RA promotes differentiation into the neural lineage. (A) Schematic protocol for neural differentiation of hPSCs. (B $\sim F$ ) Effects of hormones $(\mathrm{E} 2, \mathrm{P} 4$, and $\mathrm{DM})$ and a hormone-like molecule (RA) on the production of neural progenitors (NESTIN ${ }^{+}$and PAX6 ${ }^{+}$). (B) Flow cytometric plots showing neural progenitors at days $7 \sim 10$ following neural differentiation with or without E2, P4, DM, and RA treatment. (C) Relative levels of NESTIN ${ }^{+}$cells normalized to the control. The inset shows the percentage of NESTIN ${ }^{+}$cells. (D) Relative levels of PAX6 ${ }^{+}$cells normalized to the control. The inset indicates the frequency of PAX6 ${ }^{+}$cells. (E) Quantitative real-time PCR analysis of the neural progenitor markers NeuroD and PAX6 and the neural filament marker NF68. Gene expression is normalized to that of GAPDH. (F) Immunocytochemical analysis of $\beta$ III-tubulin from control and RA-treated cells during neural differentiation. (G) Schematic diagram of the effect of E2 and RA on lineage specification of hPSCs into hematopoietic and neural lineage. Scale bar, $100 \mu \mathrm{m}$. ${ }^{\#}$ Data are presents as the means \pm SD of three independent experiments. ${ }^{*} \mathrm{p}<0.05 ;{ }^{* *} \mathrm{p}$ $<0.01$. 
populations, which can be promoted by E2.

\section{RA promotes differentiation into the neural lineage}

To further understand the roles of hormones and hormone-like molecules in controlled cell fate decisions, we evaluated neural lineage specification. Following previously optimized protocols (Fig. 4A), we generated neurospheres, representing neural progenitors, under different conditions and examined their neural differentiation potential by comparing the expression of NSC markers, NESTIN and PAX6. In contrast to the hematopoietic differentiation results, neural potential was less affected by hormones, even in the presence of E2 (Fig. $4 \mathrm{~B} \sim \mathrm{D}$ ). However, RA still exhibited significantly reduced neural lineage specification, resulting in fewer NESTIN $^{+}$and PAX6 $^{+}$cells (Fig. 4B D). Since RA is one of the most well-known neural-inducing factor, triggering in vitro neural differentiation of hPSCs, we further verified its promotion of the neural lineage by analyzing neural cell-specific gene expression (Fig. 4E). In accordance with the restricted outcomes of NSC frequencies, expression of PAX6 was significantly suppressed upon RA treatment; however, upregulation of NF68 expression, neurofilament gene, was detected. Since NF68 is one of the most abundant cytoskeletal components of neurons, neural precursors under RA treatment likely further differentiated into neurons, resulting in a reduced frequency of NSCs. To more directly evaluate this possibility, we subjected differentiated neural precursors to stain with $\beta$ III-tubulin, a microtubule element found almost exclusively in neurons. As expected, significantly more $\beta$ III-tubulin ${ }^{+}$cells were observed after RA treatment (Fig. 4F), explaining the reduced number of neural precursor cells and implying that RA promoted neural cell fate by promoting cell differentiation into mature neurons. Taken together, these results provide new distinct roles of E2 and RA in the lineage-specific differentiation potential of hPSCs, where E2 is associated with efficient hematopoietic differentiation and RA activates neural lineage specification at the expense of other lineages.

\section{Discussion}

We investigated the roles of three hormones (E2, P4, and DM) and a hormone-like molecule (RA) to clarify in vivo hematopoietic and neural developmental programs using hPSCs as an in vitro developmental surrogate model. Our results demonstrated that the sex steroid hormone E2, but not P4, promoted multi-lineage potential of differentiated hematopoietic progenitors. E2 significantly en- hanced the functional capacity of progenitor cells in the CFU assay; however, the frequency and total number of hematopoietic cells $\left(\mathrm{CD} 45^{+}\right)$and progenitors $\left(\mathrm{CD} 34^{+}\right.$ $\mathrm{CD} 45^{+}$) were not significantly influenced by any of the investigated hormones. In addition, we demonstrated that $\mathrm{CD} 34^{+} \mathrm{CD} 45^{+}$cells from the $\mathrm{CD} 43^{-}$population, previously identified as non-hemogenic cells (23), exhibited colony-forming potential in the E2-treated group. This finding suggests new possible hemogenic precursors induced from the $\mathrm{CD}_{4} 3^{-}$population and further supports the potentiation of E2 on hematopoietic and progenitor cells (HSPCs). However, it does not disregard the effect of E2 on HSPC proliferation. In mice, conditional knock-out of Esr1, which encodes $\mathrm{ER} \alpha$, showed that E2 promoted proliferation of HSPCs in a cell-autonomous manner, without any effects on BM cellularity or cellular composition (4, 24). In addition, E2 improved the outcome of hematopoietic differentiation of human embryonic stem cells, yielding higher frequencies and numbers of HSPCs (25). Differences among the protocols used for hematopoiesis might have led to such distinct estrogen effects, and enhanced functional capacity versus proliferation of HSPCs. It is difficult to determine the exact mechanism of E2 during the whole process of hematopoietic differentiation, because $\mathrm{ER} \alpha$ is highly expressed in both hPSCs and HSPCs (4, 26). Therefore, more complex differentiation protocols with detailed treatment conditions, including treatment time and dose, are necessary to further clarify the molecular mechanisms of E2-mediated hematopoietic specification and HSPC potentiation.

$\mathrm{DM}$ is known to functionally promote erythropoiesis (9); however, we did not observed any significant changes in hematopoietic fate. For the efficient generation of mature erythrocytes, distinct culture conditions with several developmental cytokines are generally used. Thus, additional studies following optimized protocols are required to clarify the functional role and mechanism of DM in the generation of specific types of mature blood cells from hPSCs. In addition, DM has been reported to affect cells in the neural lineage, including enhancing cortical GABAergic neuron differentiation (27) and promoting hippocampal neural apoptosis (28). However, DM treatment had no effect on the neural differentiation of hPSCs in this study. Our results indirectly suggest that DM has no adverse or beneficial effects on lineage specification of hPSCs, and is more likely to affect differentiated progenitors or mature neurons rather than undifferentiated hPSCs.

$\mathrm{RA}$ is a well-known morphogen that regulates stem cell self-renewal and differentiation not only in PSCs, but also 
in HSCs and NSCs. A growing number of studies have demonstrated that RA signaling is closely related to hematopoietic differentiation and maturation. In hPSCs, inhibition of RA synthesis at the late phase (days 16 21) of hematopoietic differentiation increased the HSPC fraction without a proportional change, whereas RA activation dramatically abrogated hematopoiesis (18). In contrast, it was also demonstrated that RA enhanced hematopoietic outcomes by promoting the endothelial-hematopoietic transition (29). Furthermore, RA promoted HSC development and maturation from hemogenic endothelium, isolated from E10.5 mouse embryos, via transient downregulation of Wnt signaling $(17,18,30)$. These apparently controversial effects of RA on differentiation are probably due to its action on cells, which is strictly controlled in a spatiotemporal manner between developmental and homeostatic conditions.

In this study, RA significantly impaired hematopoietic differentiation, and HSPCs derived from RA treatment exhibited a poor functional ability as progenitors. Inhibition of RA signaling before the onset of hematopoiesis plays a critical role in the enhanced hematopoietic outcome (18). Therefore, RA might have promoted ectodermal lineage specification and neural differentiation at the expense of hematopoietic differentiation, because RA signaling in both early and late stages of embryonic development is known to promote ectodermal differentiation by repressing self-renewal-associated genes (10). We also showed that RA activated neural fate decisions by promoting further differentiation of cells into mature neurons, as indicated by enhanced $\beta$ III-tubulin ${ }^{+}$cells with upregulation of NF68 expression. However, the NSC population $\left(\mathrm{NESTIN}^{+}\right.$and $\left.\mathrm{PAX6}^{+}\right)$and PAX6 expression were significantly reduced upon RA treatment. These results could be attributed to the potential for neural differentiation of RA, which pushes NSCs into neural lineages. Several retinoic acid response element (RARE)-containing genes have been identified to contribute to neural lineage differentiation and brain development, including Raldh2, Raldh3, Cyp26a1, and Cyp26c1 (31, 32). Thus, RARE-dependent neural gene induction by RA could lead to neural lineage-bias at the expense of alternative cell fates, in contrast to E2, which enhances the clonogenic potential of hematopoietic progenitor cells without restricting other lineages.

Taken together, the present study provides evidence to understand novel effects of endocrine molecules on early embryonic development using hPSCs as a surrogate model. We expect that these novel effects of hormones and hormone-like molecules on hPSC differentiation will pro- vide new opportunities to generate functional derivatives and uncover fundamental knowledge of the endocrine system and stem cell physiology.

\section{Acknowledgments}

This study was supported by grant from the KRIBB Research Initiative Program (KGM4251824), Republic of Korea.

\section{Potential Conflict of Interest}

The authors have no conflicting financial interest.

\section{Supplementary Materials}

Supplementary data including one figure can be found with this article online at http://pdf.medrang.co.kr/paper/pdf/IJSC/IJSC-12-s18137.pdf.

\section{References}

1. Zon LI. Intrinsic and extrinsic control of haematopoietic stem-cell self-renewal. Nature 2008;453:306-313

2. Wahlster L, Daley GQ. Progress towards generation of human haematopoietic stem cells. Nat Cell Biol 2016;18:11111117

3. Espin-Palazon R, Weijts B, Mulero V, Traver D. Proinflammatory signals as fuel for the fire of hematopoietic stem cell emergence. Trends Cell Biol 2018;28:5866

4. Nakada D, Oguro H, Levi BP, Ryan N, Kitano A, Saitoh Y, Takeichi M, Wendt GR, Morrison SJ. Oestrogen increases haematopoietic stem-cell self-renewal in females and during pregnancy. Nature 2014;505:555-558

5. Varshney MK, Inzunza J, Lupu D, Ganapathy V, Antonson P, Rüegg J, Nalvarte I, Gustafsson JÅ. Role of estrogen receptor beta in neural differentiation of mouse embryonic stem cells. Proc Natl Acad Sci U S A 2017;114:E10428E10437

6. Joshi PA, Jackson HW, Beristain AG, Di Grappa MA, Mote PA, Clarke CL, Stingl J, Waterhouse PD, Khokha R. Progesterone induces adult mammary stem cell expansion. Nature 2010;465:803-807

7. Canciello A, Russo V, Berardinelli P, Bernabò N, Muttini A, Mattioli M, Barboni B. Progesterone prevents epithelial-mesenchymal transition of ovine amniotic epithelial cells and enhances their immunomodulatory properties. Sci Rep 2017;7:3761

8. Nürnberg E, Horschitz S, Schloss P, Meyer-Lindenberg A. Basal glucocorticoid receptor activation induces proliferation and inhibits neuronal differentiation of human induced pluripotent stem cell-derived neuronal precursor cells. J Steroid Biochem Mol Biol 2018;182:119-126

9. Narla A, Dutt S, McAuley JR, Al-Shahrour F, Hurst S, McConkey M, Neuberg D, Ebert BL. Dexamethasone and 
lenalidomide have distinct functional effects on erythropoiesis. Blood 2011;118:2296-2304

10. Rhinn M, Dollé P. Retinoic acid signalling during development. Development 2012;139:843-858

11. Tagliaferri D, De Angelis MT, Russo NA, Marotta M, Ceccarelli M, Del Vecchio L, De Felice M, Falco G. Retinoic acid specifically enhances embryonic stem cell metastate marked by Zscan4. PLoS One 2016;11:e0147683

12. Maden M. Retinoic acid in the development, regeneration and maintenance of the nervous system. Nat Rev Neurosci 2007;8:755-765

13. Addae C, Yi X, Gernapudi R, Cheng H, Musto A, Martinez-Ceballos E. All-trans-retinoid acid induces the differentiation of encapsulated mouse embryonic stem cells into GABAergic neurons. Differentiation 2012;83:233-241

14. Gupta S, Sivalingam D, Hain S, Makkar C, Sosa E, Clark A, Butler SJ. Deriving dorsal spinal sensory interneurons from human pluripotent stem cells. Stem Cell Reports 2018;10:390-405

15. Podleśny-Drabiniok A, Sobska J, de Lera AR, Gołembiowska K, Kamińska K, Dollé P, Cebrat M, Krężel W. Distinct retinoic acid receptor (RAR) isotypes control differentiation of embryonal carcinoma cells to dopaminergic or striatopallidal medium spiny neurons. Sci Rep 2017;7: 13671

16. Cabezas-Wallscheid N, Buettner F, Sommerkamp P, Klimmeck D, Ladel L, Thalheimer FB, Pastor-Flores D, Roma LP, Renders S, Zeisberger P, Przybylla A, Schönberger K, Scognamiglio R, Altamura S, Florian CM, Fawaz M, Vonficht D, Tesio M, Collier P, Pavlinic D, Geiger H, Schroeder T, Benes V, Dick TP, Rieger MA, Stegle O, Trumpp A. Vitamin A-retinoic acid signaling regulates hematopoietic stem cell dormancy. Cell 2017;169: 807-823.e19

17. Collins SJ. The role of retinoids and retinoic acid receptors in normal hematopoiesis. Leukemia 2002;16:1896-1905

18. Rönn RE, Guibentif C, Moraghebi R, Chaves P, Saxena S, Garcia B, Woods NB. Retinoic acid regulates hematopoietic development from human pluripotent stem cells. Stem Cell Reports 2015;4:269-281

19. Lee JH, Lee JB, Shapovalova Z, Fiebig-Comyn A, Mitchell RR, Laronde S, Szabo E, Benoit YD, Bhatia M. Somatic transcriptome priming gates lineage-specific differentiation potential of human-induced pluripotent stem cell states. Nat Commun 2014;5:5605

20. Lee JH, Salci KR, Reid JC, Orlando L, Tanasijevic B, Shapovalova Z, Bhatia M. Brief report: human acute myeloid leukemia reprogramming to pluripotency is a rare event and selects for patient hematopoietic cells devoid of leukemic mutations. Stem Cells 2017;35:2095-2102
21. Angelos MG, Abrahante JE, Blum RH, Kaufman DS. Single cell resolution of human hematoendothelial cells defines transcriptional signatures of hemogenic endothelium. Stem Cells 2018;36:206-217

22. Vo LT, Kinney MA, Liu X, Zhang Y, Barragan J, Sousa PM, Jha DK, Han A, Cesana M, Shao Z, North TE, Orkin $\mathrm{SH}$, Doulatov S, Xu J, Daley GQ. Regulation of embryonic haematopoietic multipotency by EZH1. Nature 2018;553: 506-510

23. Vodyanik MA, Thomson JA, Slukvin II. Leukosialin (CD43) defines hematopoietic progenitors in human embryonic stem cell differentiation cultures. Blood 2006;108: 2095-2105

24. Illing A, Liu P, Ostermay S, Schilling A, de Haan G, Krust A, Amling M, Chambon P, Schinke T, Tuckermann JP. Estradiol increases hematopoietic stem and progenitor cells independent of its actions on bone. Haematologica 2012;97: 1131-1135

25. Kim HR, Lee JH, Heo HR, Yang SR, Ha KS, Park WS, Han ET, Song H, Hong SH. Improved hematopoietic differentiation of human pluripotent stem cells via estrogen receptor signaling pathway. Cell Biosci 2016;6:50

26. Hong SH, Nah HY, Lee YJ, Lee JW, Park JH, Kim SJ, Lee JB, Yoon HS, Kim CH. Expression of estrogen receptor-alpha and -beta, glucocorticoid receptor, and progesterone receptor genes in human embryonic stem cells and embryoid bodies. Mol Cells 2004;18:320-325

27. Baud O, Verney C, Evrard P, Gressens P. Injectable dexamethasone administration enhances cortical GABAergic neuronal differentiation in a novel model of postnatal steroid therapy in mice. Pediatr Res 2005;57:149-156

28. Spreer A, Gerber J, Hanssen M, Schindler S, Hermann C, Lange P, Eiffert H, Nau R. Dexamethasone increases hippocampal neuronal apoptosis in a rabbit model of Escherichia coli meningitis. Pediatr Res 2006;60:210-215

29. Yu C, Liu Y, Miao Z, Yin M, Lu W, Lv Y, Ding M, Deng $H$. Retinoic acid enhances the generation of hematopoietic progenitors from human embryonic stem cell-derived hemato-vascular precursors. Blood 2010;116:4786-4794

30. Chanda B, Ditadi A, Iscove NN, Keller G. Retinoic acid signaling is essential for embryonic hematopoietic stem cell development. Cell 2013;155:215-227

31. Halilagic A, Ribes V, Ghyselinck NB, Zile MH, Dollé P, Studer M. Retinoids control anterior and dorsal properties in the developing forebrain. Dev Biol 2007;303:362-375

32. Uehara $M$, Yashiro K, Mamiya S, Nishino J, Chambon P, Dolle P, Sakai Y. CYP26A1 and CYP26C1 cooperatively regulate anterior-posterior patterning of the developing brain and the production of migratory cranial neural crest cells in the mouse. Dev Biol 2007;302:399-411 